\title{
Utilisation du principe de checklists pour l'élaboration de procédures facilitant le développement du savoir- faire dans les projets de conception
}

\author{
François Charron et Jean-Philippe Desbiens \\ Département de génie mécanique, Université de Sherbrooke \\ 2500, boul. de I'Université, Sherbrooke (Québec), J1K 2R1
}

\section{Résumé}

Le Département de génie mécanique de l'Université de Sherbrooke a introduit des projets majeurs de conception dans son baccalauréat depuis 1992. C'est d'ailleurs en s'inspirant des processus modernes de développement de produit (PDP) dont celui de l'ingénierie simultanée que le Département de génie mécanique a développé son propre PDP considérant les objectifs pédagogiques d'un projet majeur de conception réalisé par des étudiants et aussi les contraintes associées à un tel projet dans le cadre du baccalauréat. Ce PDP débute par la définition du projet de conception et il se termine par la validation expérimentale d'un prototype fonctionnel. La réalisation des projets majeurs de conception nécessite la mise en œuvre du savoir, du savoir-faire et du savoir-être des étudiants.

Hors, la formation par compétence en génie mécanique à l'Université de Sherbrooke intègre le développement du savoir (sciences du génie, mathématiques, etc.), du savoir-faire (conception, mécatronique, entrepreneuship, etc.) et du savoir-être (travail 
en équipe, professionnalisme, etc.). Évidemment, bien que le savoir soit documenté dans plusieurs livres, il en va tout autrement pour le savoir-faire dont le savoir procédural qui est de nature souvent plus tacite et qui nécessite une vue globale de plusieurs éléments. Néanmoins, les activités des projets majeurs de conception font appel au savoir-faire lors de diverses activités: écriture de documents techniques, procédures de calculs analytiques et numériques, analyses par éléments finis, création de modèle virtuel $\mathrm{CAO}$, mise en plan de pièces et assemblages, etc. Malheureusement, le savoir procédural associé à ces activités est généralement abordé rapidement dans le curriculum faute de temps et d'ouvrages de référence pour l'enseigner. Pour cette raison, les compétences des étudiants sont souvent déficientes pour réaliser certaines activités des projets de conception selon les règles de l'art (ex. produire des dessins de fabrication de pièces mécaniques). De plus, certaines de ces activités font appel à un niveau de compétence important et sont d'une complexité qui dépasse parfois le cadre des notions vues en classe (ex. mise en plan d'un étau vs. mise en plan d'une motoneige). Ainsi, afin d'aider les étudiants à développer des compétences où le savoir procédural est important, la notion de checklists d'activités a été exploitée pour développer de courts documents d'aide que l'on appellera « procédure » puisque ces dernières documentent les façons de faire pour réaliser une activité donnée selon les règles de l'art.

Ainsi, le concept de checklist utilisé ici se dissocie quelque peu des listes de vérification habituelles communément utilisées dans plusieurs domaines. En effet, la checklist utilisée dans le contexte des projets de conception vise davantage à servir de 
guide dans la réalisation d'une activité liée au savoir-faire plutôt que de vérifier la présence d'un objet, d'un résultat ou d'une activité. Cette dernière est essentiellement une liste d'énoncés qui permet de vérifier si tous les aspects d'une activité ont été couverts et réalisés correctement. Elle permet donc d'expliciter un savoir tacite autrement difficile à transmettre aux étudiants d'une façon efficace. Évidemment, les éléments constituants les checklists véhiculent l'information ou les références nécessaires aux étudiants pour favoriser leur auto-apprentissage.

Certaines checklists (procédures) sont désormais disponibles dans la section «Ressources pour les projets» du site Internet du génie de la conception (www.conception.gme.usherb.ca). 